\title{
Cardiovascular data analysis using electronic wearable eyeglasses - preliminary study
}

\author{
Adam Bujnowski, Jacek Ruminski, Mariusz Kaczmarek, Krzysztof Czuszynski, Piotr Przystup \\ Gdansk University of Technology \\ ul. Narutowicza 11/12, 80-233 Gdansk, Poland \\ Email: bujnows@biomed.eti.pg.gda.pl
}

\begin{abstract}
The paper presents an alternative approach to the monitoring of the cardiovascular system. The study depicts configurations of the utilized system and preliminary results of electrical and mechanical parameters of the cardiac system which can be measured using a head-worn device.
\end{abstract}

\section{INTRODUCTION}

$\mathbf{T}$ HE continuously progressing miniaturization in modern electronics results in the creation of miniature, yet powerful devices that can be battery powered and network connected. Many of such devices are wearable. The permanently increasing computational power, characterised by a reasonable runtime and fitted with batteries, creates new possibilities.

Wearable electronic eyeglasses can be regarded as an example of such devices. In fact, the eyeglasses are a miniaturized computer that fits in the frame resembling traditional glasses. Many of such devices are available - equipped with various configurations. Most of them have a local near to eye display. These eyeglasses can be either semi-transparent or completely isolating (non-transparent). Most of these electronic glasses are equipped with a single camera for observing the front scene in relation to the person wearing the device.

A major disadvantage of the majority of the commercially available platforms is the limited potential for future expansion. In fact, most of the modifications are limited to writing new applications that will utilize the hardware available on the platform or the hardware wirelessly connected by means of Wi-Fi or Bluetooth interfaces [6], [7].

As a result of the ERA-NET-CHIST-ERA II project The interactive eyeglasses for mobile, perceptual computing (eGlasses) a new open platform in the form of the glasses was designed and developed [1]. It is considered as an open platform that can be extended by means of adding local hardware using various interfaces.

The eGlasses consist of a powerful computer running the Linux or Android system. Expansions might regard various aspects of the device's usage. As an example, an Infrared camera allows them to operate in the darkness or can help the user to estimate the object's temperature. The eyetracker camera enables user interface navigation in a hands-free mode

This work has sponsored by the ERA-NET-CHIST-ERA II project The interactive eyeglasses for mobile, perceptual computing (eGlasses) and partly from Gdansk University if Technology - Faculty of Electronics, Telecommunications and Informatics statue funds.
[2]. Additional proximity sensors might be used for obstacle detection [3].

One type of possible platform extensions are biomedical measuring units for measuring biosignals such as ECG, EEG, EMG, and the body temperature of the person wearing such a platform [4].

This paper focuses on the measurement of the cardiovascular system. The measurement of the electrical biopotentials from the cardiac muscle (ECG) is usually measured from the contacting electrodes located on the chest - close to the cardiac muscle. A wearable platform such as eGlasses should not be large nor complicated in application. We are limit measuring electrodes location to close to the ordinary glasses frame. There exist known works related to the estimation of the cardiac potentials with ballistic data collected on the head [8].

This paper is organized as follows. In section II, the eGlasses platform is briefly discussed and an experimental set-up is proposed. Section III presents the results of measurements, and section IV outlines the conclusions.

\section{Materials And Methods}

\section{A. The eGlasses platform}

The eGlasses are designed to be expandable. They consist of three major boards referred to as the "base board", "side board" and "T-board". The connection between the boards is made using flat flexible connectors (FFC) and is shown in Fig. 1. The main unit is the base board. Currently, it is based on the DART4460 board from Variscite [5]. The board is based on the dual-core OMAP 4460 with 1GB RAM and internal FLASH memory (8GB). The DART 4460 module is located on the larger board where level converters, position sensors, DC-DC power sources and peripheral connectors are located. In the future, this board might be replaced by a more powerful one. There is a QUAD core board with IMX6 SoC on board in its preparatory stage. The main board is designed to be located in the right panel of the eGlasses. On the opposite side - the right panel - there is a battery holder with an additional board carrying mainly the USB hub with a USB-to-serial converter. In the front panel, in the centre, the T-shaped board carrying the front camera is located. This board additionally holds the IR camera and is used as an interconnection for signals between the base board and the side board.

The major advantage of the eGlasses platform is the amount of available IO ports. User can have several USB ports, 


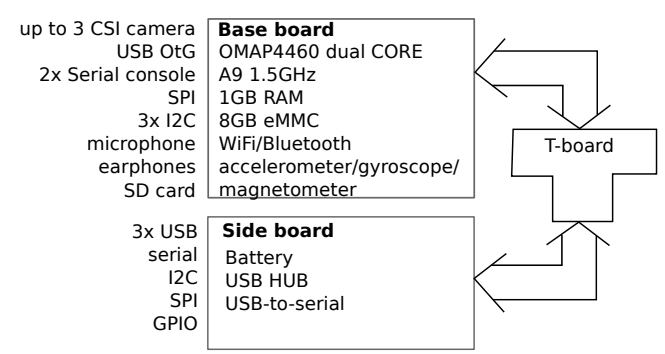

Fig. 1. Block diagram of the eGlasses platform

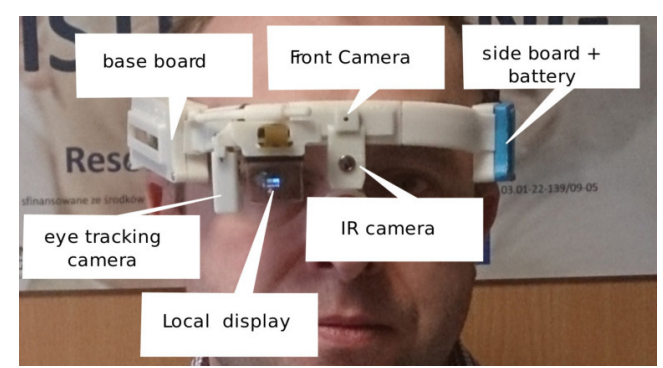

Fig. 2. The eGlasses and location of major components

serial, SPI, I2C and USB ports for interfacing with additional extensions. The eGlasses prototype is shown in fig. 2.

\section{B. Cardiac measurements}

Self-diagnostics can play an important role in several fields of life. One of the most frequently explored types of vital systems are those related to the cardiovascular system. There is a variety of different methods for cardiac measurements. One of the simplest and cheapest techniques is an electrical cardiac potentials measurement. The ECG signal is generated by dedicated cell groups inside the heart. Outside it can be regarded as a current dipole. A conventional ECG test measures a difference of potentials between specific locations on the chest. In the case of the eGlasses we raise the question if it is possible to measure the ECG signal on the head. Therefore, we have selected an electrode location on the both sides of the face in front of the ears. In order to improve the CMRR, the ECG measurement system uses a dedicated electrode that returns the inverted common potential back to the body, i.e. the DRL (Driven Right Leg) solution is utilized. A middle point between measurements electrodes was selected as the location of the DRL electrode (Fig. 3). The ECG signal observed is characterised by a weak amplitude. In order to measure such a signal, we have designed a custom single channel ECG measurement system with five times greater gain when compared to an ordinary chest-based system.

Another signal coming out of the cardiac system is blood pulsation in vessels. A piezoelectric transducer was used to measure blood pulsation. We have used a temporal artery to access the mechanical pulsation. Moreover, the optimal position of the electrodes has been tested. We have prepared one more module, allowing simultaneous measurements of four differential signals. The first channel has been used for

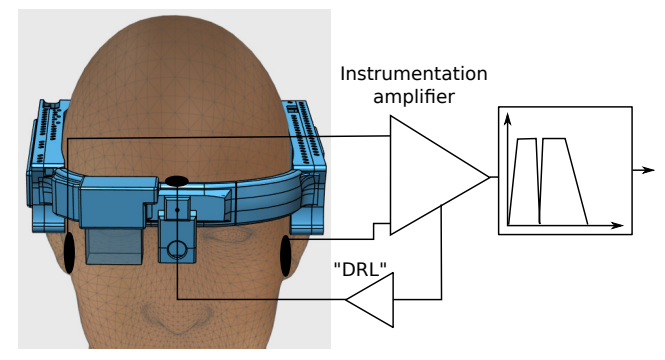

Fig. 3. Electrode's location for the ECG measurement using eGlasses

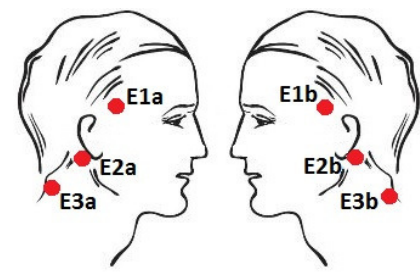

Fig. 4. ECG electrode's placement on the head

traditional ECG measurement, thus two electrodes were placed on the examined person's chest. The other three channels were used to measure the ECG on the head. Electrodes were placed on the temples (ECG1), behind the ears (ECG2) and on the neck (ECG3). Signals were recorded with 24-bit resolution and $250 \mathrm{~Hz}$ sampling rate. Each channel has been filtered with a $50 \mathrm{~Hz}$ notch filter in digital domain. The base line drift has been removed using a median filter (Fig. 4).

\section{Experiment setup}

In order to conduct the experiment, we have prepared two modules of ECG and a piezoelectric sensor (Fig. 5). The module marked as ECG1 with ordinary amplification was used for the chest as a reference module, and ECG2 with five times greater amplification was used for head measurements. We have used a piezoelectric sensor and a simple amplifier. The signals were connected to the digital storage oscilloscope and recorded on a USB stick. We have collected data from five volunteers aged 22-46 to prove the system's performance. To acquire ECG signals, standard adhesive and disposable electrodes were used.

\section{RESULTS}

An experimental set-up has been created on the prototyping bread-board (Fig. 6). The ECG1 and ECG2 amplifiers were assembled on separate printed circuit boards (PCB). The piezoelectric transducer amplifier was assembled using trough hole components located directly on the bread-board. All operational amplifiers enabled the operation from a single power supply. We used a 5V DC power supply with medical class separation from the mains.

As a piezoelectric transducer we have used FT-27T-4.0A transducer with $4 \mathrm{kHz}$ resonant frequency, $200 \Omega$ of resonant resistance and $25 \mathrm{nF}$ capacitance. The unfiltered signals were 


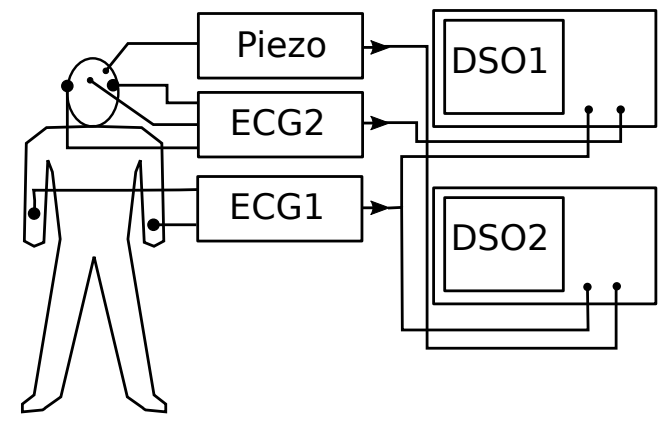

Fig. 5. Block diagram of the experiment setup

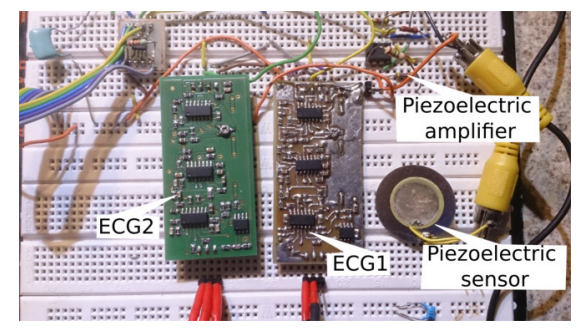

Fig. 6. ECG measurement boards

connected to two digital storage oscilloscopes (DSO), simultaneously recording data (Fig. 7). In order to synchronize the data streams, we connected output of the ECG1 signal to both instruments. It allowed us to estimate the time shift between the instruments.

Based on the raw data, one might notice a similarity between the ECG recorded using the standard procedure and the signal obtained from the head. Additionally, the data recorded from the pulsating vessel resembles arterial pulse (Fig. 8). The signals recorded reflect the standard Wiggers diagram, where atrial pressure peaks are in relation to the ECG signal. In Fig. 8, T-peaks of the electrocardiogram are coinciding with the arterial pressure peaks related to the opening of the mitral valve.

The relations between the ECG signals recorded by elec-
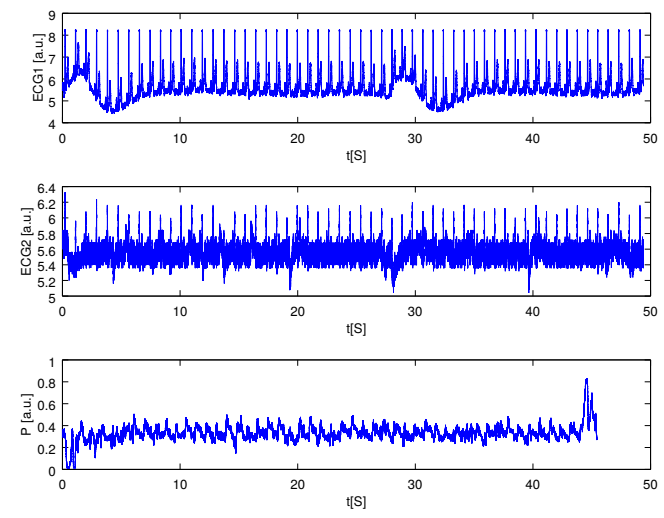

Fig. 7. Results of measurements - raw signals from the top: ECG signal from the chest, ECG signal measured on the head and piezoelectric pulse

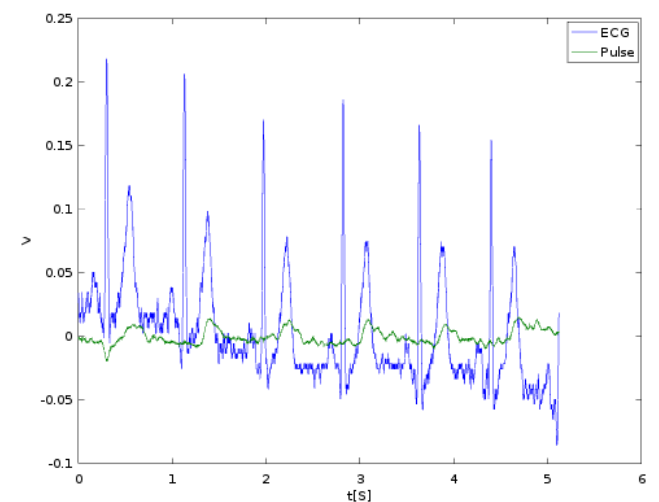

Fig. 8. Results of measurements - ECG data with recorded pulse signal measured by the piezo sensor

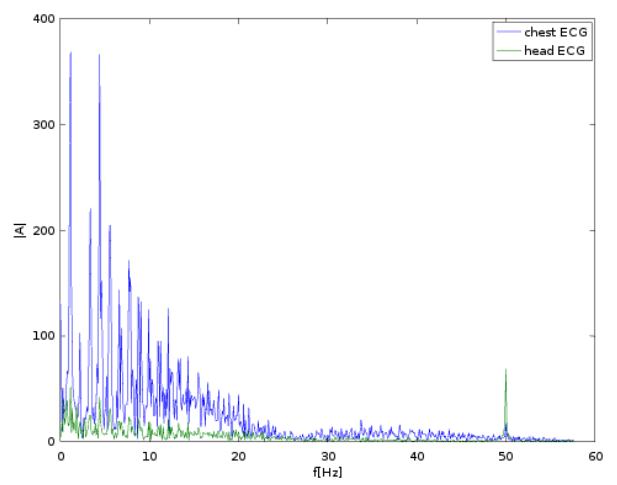

Fig. 9. Simultaneous measurements of the chest ECG with ECG recorded from the head spectrum of modulus

trodes located in different places on the body (chest and head) were examined (Fig. 11). The amplification of the head ECG is about five times greater than the chest ECG. Moreover, the recorded QRS amplitude is much smaller for the headmeasured signal.

The components of both signals were examined by means of the Fourier transformation (Fig. 9). The spectrum of the signals was restricted to $60 \mathrm{~Hz}$. The spectrum data showed a peak of the component at $50 \mathrm{~Hz}$. After filtration, the $50 \mathrm{~Hz}$ component disappeared and simultaneously the head ECG became more "clear" (Fig. 10). When analysing the signal from Fig. 10, QRS peaks are visible and the heart-rate can be calculated, however, the ratio of the detected QRS periods is about $10 \%$ smaller than that from the signal acquired on the chest.

Finally, the optimum position of the electrodes has been selected in order to obtain the highest amplitude of the QRS complex (Fig. 11). The amplitudes of the QRS complexes for the examined leads can be found in Tab. I.

\section{CONCLUSION}

The measurement of the ECG signal using electrodes located on the head is possible, however, a custom design of the acquisition system should be prepared. The ECG signal 
TABLE I

AVERAGE QRS COMPLEX AMPLITUDE MEASURED ON THE VARIOUS POSITION ON THE HEAD

\begin{tabular}{|l|c|c|c|c|}
\hline & Chest ECG & ECG1 & ECG2 & ECG3 \\
\hline QRS amplitude & $650 \mu V$ & No QRS & $30 \mu V$ & $30 \mu V$ \\
\hline
\end{tabular}

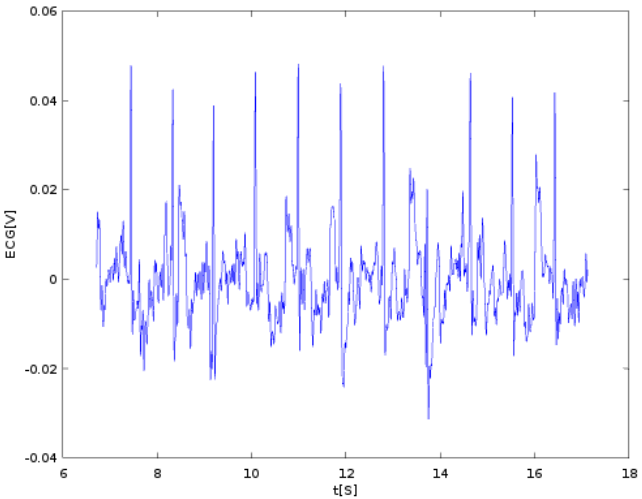

Fig. 10. Low-pass filtered ECG signal measured on the head (bandwidth $0.05-30 \mathrm{~Hz})$

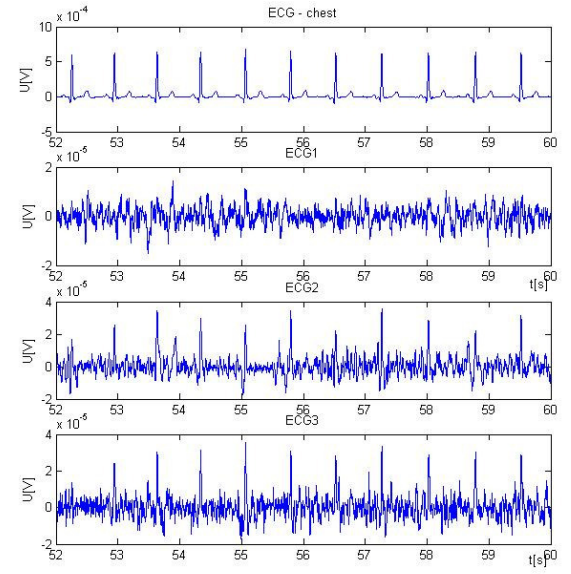

Fig. 11. ECG signals recorded on chest (upper plot) and on the head (ECG1-3)

measured on the head is nosier, but it is still possible to recognize the QRS periods and calculate the heart rate.

It is not possible to acquire the proper ECG signal from any electrode combination over the head. A detailed analysis should be performed to choose the best electrodes set-up.
A preliminary study shows that it is possible to record the QRS complex using electrodes located on the neck, or behind the ears. However, the recorded signal is approximately 20 times smaller than the signal measured in a conventional way. Assuming that the ECG will be measured using the eGlasses platform, the preferable position of the electrodes is just behind the ears. In this case, the electrodes could be embedded in the earpiece of the glasses.

Additionally, it is possible to measure the blood pulsation component on the head using a temporal artery. Unfortunately, the localization of this artery on the head is difficult. In the study presented, traditional disposable and adhesive ECG electrodes were used. In the case of a wearable platform, longterm electrodes should be considered.

\section{REFERENCES}

[1] Homepage of the eGlasses project; http://www.e-glasses.net [accessed 15.03.2016]

[2] Kocejko, T., Ruminski, J., Wtorek, J., Martin, B., Eye tracking within near - to - eye display, in Human System Interactions (HSI), 2015 8th International Conference on, pp.166, - 172, 25 - 27 June 2015, doi: 10.1109/HSI.2015.7170661

[3] Bujnowski, A.,Czuszynski, K., Ruminski, J., Wtorek, J., McCall, R., Popleteev, A., Louveton, N., Engel, T., Comparison of active proximity radars for the wearable devices, in Human System Interactions (HSI), 2015 8th International Conference on , pp.158 - 165, 25 -27 June 2015 doi:10.1109/HSI.2015.7170

[4] Bujnowski A., Ruminski J., Przystup P., Czuszynski K., Kocejko T. Self Diagnostics Using Smart Glasses - Preliminary Study, in Human System Interactions (HSI), 2016 9th International Conference on (accepted)

[5] The DART4460 specification http://www.variscite.com/products/ system-on-module-som/cortex-a9/dart-4460-cpu-ti-omap-4-omap4460 [accessed 14.03.2016]

[6] J. Ruminski, M. Smiatacz, A. Bujnowski, A. Andrushevich, M. Biallas, and R. Kistler, Interactions with recognized patients using smart glasses, in Proceedings of the 8th International Conference on Human System Interactions (HSI 15), pp. 187194, June 2015.

[7] J. Ruminski, A. Bujnowski, J. Wtorek, A. Andrushevich, M. Biallas, and R. Kistler, Interactions with recognized objects, in Proceedings of the 7th International Conference on Human System Interactions (HSI 14), pp. 101105, Costa da Caparica, Portugal, June 2014.

[8] D. Da He, E. S. Winokur and C. G. Sodini, ”A continuous, wearable, and wireless heart monitor using head ballistocardiogram (BCG) and head electrocardiogram (ECG)", Engineering in Medicine and Biology Society, EMBC, 2011 Annual International Conference of the IEEE, Boston, MA, 2011, pp. 4729-4732. 